\title{
Reduction of Rank-Reduced Orientation-from-Color Problem with Many Unknown Lights to Two-Innage Known-Illuminant Photometric Stereo
}

\author{
Mark S. Drew \\ School of Computing Science, Simon Fraser University \\ Vancouver, B.C. Canada V5A 1S6 \\ e-mail mark@cs.sfu.ca
}

\begin{abstract}
How does shape-from-shading proceed when two or more differently-colored light sources are present? For a uniformly colored Lambertian surface illuminated by a collection of point or extended light sources or interreflections, with unknown directions and strengths, illumination varies spectrally with orientation from the surface. If light varies enough in color and direction, then surface orientation can be recovered by a kind of photometric stereo, but with a single image and many unknown lights. RGB values lie on an ellipsoid in color space, and the strengths and directions of three effective "lights" can be recovered from regression and by additional constraints derived from three estimates of light source tilt.

However, it is quite likely that illumination color does not vary enough. In that case $R G B$ points fill a planar $2 \mathrm{D}$ ellipse in color space. Here we give a result for the ellipse boundary that enables one to recover the strengths and angle between two effective lights producing the $2 \mathrm{D}$ shading. We then show that equations for the tilts of the linearly related three effective lights in 3D give an additional three independent constraints. Solving yields lights in 2D and hence in 3D as well. Thus the rank-2 orientation-from-color problem reduces to known-light 2 -image photometric stereo. Robust methods are used throughout.
\end{abstract}

\section{Introduction}

On a clear day the shape-from-shading problem is complicated by light from at least two different directions and of two different colors as well - light from the sun and sky light. Brooks and Horn [1] address this problem by explicitly incorporating a sky radiation component in an iterative scheme for finding shape and point source light direction for a Lambertian surface. For the sky light they simply take the overall direction as toward the $z$-axis. This makes sense because of the well-known fact that Lambertian surfaces sum up an extended light source into an equivalent point source, although some greater care is required in correctly calculating the sky direction ([9], p.237).

Nonetheless, the color information in the two sources of illumination is not put to use. If one could effectively take apart the shading from the two different light sources, then recovery of the two components of normalized surface orientation vectors could be addressed using 2-image photometric stereo, a problem whose solution is known $[12,11,21]$.

Of course, a real image is further complicated by specularities, shadows, interreflected light, and points dent radiation is comprised of many unknown lights that are far enough apart and also vary spectrally with direction from the surface, then for a single Lambertian surface under conditions of orthography the situation is reducible to that of 3 -image photometric stereo $[18,19]$, even if the directions, strengths, and colors of the collection of many lights is unknown $[3,10,15,6,4,2]$. For in this case the scatterplot of RGB points in color space lie on an ellipsoid. Surface normals are simply a linear transform away from colors, for points that see all lights. The linear transform is made up of three effective lights.

A successful way to deal with outliers, such as shadows and specularities, is to find the Lambertian ellipsoid using robust regression. Then the outliers arise from two sources: points that do not see all lights, and points that are specular, and these outliers are identified by the robust regression [4]. (For specular detection to succeed, $50 \%$ plus 1 of the pixels must be near-Lambertian.)

However, the color space ellipsoid for unknown lights results in surface normals recovered only up to an overall orthogonal transformation. One method to recover the orthogonal transformation along with surface normals is to employ an integrability condition on recovered derivatives $p(x, y), q(x, y)[10,6]$. However, that method is subject to possible reversed direction of recovered normals, since the orthogonal transformation may well contain an axis reversal. A simpler method that avoids this problem is to use the robustly recovered ellipsoid to establish the three strengths of and the angles between the effective lights. Then Pentland's [13] or some other light-source-direction finder can be used to calculate the effective lights' tilt angles (ignoring the often incorrect slant angles produced by the light-source-direction finder). The three angles between pairs of normalized effective lights then provide three constraints on those lights' slant angles [2]. Once the lights are recovered the surface normals may be recovered by photometric stereo.

The color space ellipsoid is a direct extension of an idea due to Woodham et al. [20] for finding unknown (fixed) lights for each of three graylevel images, with lights illuminating the object in turn. The extension uses an observation originally due to Petrov [14] that for a Lambertian surface illuminated by lights that vary spectrally with orientation from the surface, normal vectors are a linear transformation, $\boldsymbol{F}$, say, away from colors. The issue of the rank of $F$ is an important one. In [15] it is shown that for a $\operatorname{rank}^{-3}$ surface (i.e., not degenerate in some way, e.g., not a cylinder) then for a full rank (i.e., rank-3) matrix $\boldsymbol{F}$ colors lie on an ellipsoid in color space and one can recover normals from colors. However, as first observed in [3, 4], the rank of $\boldsymbol{F}$ arises from the rank of its factors, made up of a color matrix $\boldsymbol{B}$ and a light-direction matrix $\boldsymbol{A}$. In 
other words, for this orientation-from-color problem to be equivalent to 3-image photometric stereo (with unknown lights, one Lambertian surface), the collection of spectrally-varying colors must be linearly independent (rank-3) and so must the set of light-source directions.

However, while it is reasonable to expect a rank-3 surface one cannot be so sure about a rank-3 lighting environment. For if a surface were illuminated by (possibly many) lights that were too close in color then colors may lie on a 2-dimensional surface in color space even if the lights are widely separated in space.

In [15] it is stated that in such a situation colors should lie within a flled ellipse in color space. However, it is not obvious what should be the analytic description of such a filled ellipse. Below, colors that are rank-2 are first transformed to 2-space color coordinates. Carrying out a robust estimation of the plane normal allows elimination of shadowed (or specular) points as outliers. Then, a new result is derived giving the equation of the ellipse boundary in terms of matrix $\boldsymbol{F}$. From this it is possible to state the strengths of and single angle between two effective lights in the colorspace plane. However, this still leaves an arbitrary 3-dimensional orthogonal transformation unknown.

To further constrain matrix $\boldsymbol{F}$ we must go back to 3 -space. From each of the three RGB color bands we can calculate the tilt angle using Pentland's or another light-direction finder. We show that these form three independent constraints on matrix $\boldsymbol{F}$ provided the set of object normal vectors has diagonal correlation matrix (as would be the case for a surface with uniformly distributed normals). For the (normalized) effective light vectors in 2-space we now have one constraint from the dot-product, and three more from RGB light directions. The resulting equations produce a set of two solutions; only one has the correct $3 \mathrm{D}$ tilt angles.

In test images we found that $\mathrm{rank}-2$ lighting is far more common in ordinary lighting environments than rank-3 lighting or, for that matter, rank-1 lighting. Therefore the analysis presented here is required.

In $\$ 2$, the linear relation between normals and colors is recapitulated, for a 3-dimensional lighting environment. The rank-reduced case is set out in $\$ 3$. In $\$ 4$ extra constraints are introduced from tilts in three dimensions. In $\$ 5$ a typical real image is studied in detail. Recovery of depth is seen to be reasonable.

\section{Orientation-from-Color}

For a camera system with RGB optical response functions $Q(\lambda)$, for a single Lambertian surface with surface normals $\boldsymbol{n}$ the measured RGB color $\boldsymbol{\rho}$ is

$\boldsymbol{\rho}=\sum_{i=1}^{L}\left(\boldsymbol{a}_{i}^{T} \boldsymbol{n}\right) \int E_{i}(\lambda) S(\lambda) \boldsymbol{Q}(\lambda) d \lambda \quad(1)$ luminated by a (possibly large) set of $L$ lights, e.g. a discretized set of extended or point light sources. Here we assume conditions of distant viewing and distant lighting from lights with normalized direction vectors $\boldsymbol{a}_{i}$ and spectral power distributions $E_{i}(\lambda)$, and integrate over the visible spectrum.

When reflected from the surface, each of the $i$ lights produces a color

so that eqn.(1) $\boldsymbol{b}_{i} \equiv \int E_{i}(\lambda) S(\lambda) \boldsymbol{Q}_{(3)}(\lambda) d \lambda$,

$\boldsymbol{B} \boldsymbol{B} \boldsymbol{A}$

with $L \times 3$ direction matrix $\boldsymbol{\rho} \boldsymbol{A}^{\prime}$ and $3 \times L$ color strength matrix $\boldsymbol{B}: \boldsymbol{A}=\left(\boldsymbol{a}_{1}, \boldsymbol{a}_{2}, \ldots \boldsymbol{a}_{L}\right)^{T}$, $\boldsymbol{B}=\left(\boldsymbol{b}_{1}, \boldsymbol{b}_{2}, \ldots \boldsymbol{b}_{L}\right)$.
Letting $\boldsymbol{F}^{T} \equiv \boldsymbol{B} \boldsymbol{A},(3)$ expresses a linear relationship between normals and colors:

$$
\rho=\boldsymbol{F}^{T} \boldsymbol{n} \text {. }
$$

In terms of the $N \times 3$ collection $\mathcal{R}$ of colors for all $N$ pixels and the $N \times 3$ collection $\mathcal{N}$ of normal vectors we have

$$
\mathcal{R}=\boldsymbol{F} \mathcal{N}
$$

Pixels not obeying the relationship (4a) are those that see a different collection of lights from all those present, because of shadowing, or pixels that do not obey the Lambertian rule sufficiently closely.

Matrix $\boldsymbol{F}$ is that matrix expressing the image irradiance equation for most of the pixels. A regression finds $\boldsymbol{F}$ with confidence provided most pixels see most lights. However, it is not the case that $\boldsymbol{F}$ is always invertible. In particular, for a rank-2 color matrix $\boldsymbol{B}$ matrix $\boldsymbol{F}$ must also be rank-2. In that case the best we can hope for is a reduction of the problem to that of 2-image photometric stereo.

In the full-rank case one can find matrix $\boldsymbol{F}$ as follows. Denoting the inverse of $\boldsymbol{F}^{T}$ by $\boldsymbol{G}$, we have

$$
\boldsymbol{n}=\boldsymbol{G} \boldsymbol{\rho} \text {. }
$$

Since $\boldsymbol{n}$ is unit length, $\rho$ is constrained to lie on an ellipsoid centered on the origin in color space:

$$
1 \equiv n^{T} n=\rho^{T} G^{T} G \rho \equiv \rho^{T} C \rho
$$

Since $\boldsymbol{C}$ is $\boldsymbol{G}^{T} \boldsymbol{G}$, it is $3 \times 3$ and symmetric positive definite with 6 independent elements; it determines $\boldsymbol{F}$ up to an orthogonal transformation. Eqn. (6) can be considered a regression with explanatory variables $\left(\rho_{1}^{2}, \rho_{2}^{2}, \rho_{3}^{2}, 2 \rho_{1} \rho_{2}, 2 \rho_{1} \rho_{3}, 2 \rho_{2} \rho_{3}\right)$ and all right-handsides equal to 1 (cf. the Appendix to [19], which deals with three separate images and therefore has no matrix $\boldsymbol{B}$, or [4], which does have a $\boldsymbol{B}$ ).

\section{Rank-reduced}

\section{Orientation-from-color}

To recover surface orientation vectors $\boldsymbol{n}$ we can begin by transforming RGB color space vectors into a coordinate system in the 2-dimensional surface in which vectors $\rho$ lie.

To make the following discussion clearer, consider Fig.1(a), an exemplar image formed by synthetically shading a range image. Suppose matrix $\boldsymbol{A}$ stores the directions to five lights, with tilt and slant angles (azimuth and colatitude - zenith angle - in degrees) of $(24,39),(156,39),(0,0),(-24,39),(-156,39)$. In order to use real lights and surfaces, here "light skin" was used as surface reflectance function and five standard (all fairly white) illuminants were used: illuminants $A, B, C, D 65$, and FL, representing incandescent and fluorescent light and various sunlights. The camera response curves for a Sony DXC151 camera with infrared filter were used. Lambertian shading for each light with self-shadowing was calculated using a ray-tracing algorithm, with summed $\max (\|\rho\|)=255$. Additive Gaussian noise was then added with rms value $1 / 255$, resulting in Fig.1(b) (displayed in black and white). Results for other synthetic images were very similar to those reported below for this exemplar.

Now consider the rank of matrix $\vec{F}$. Firstly, the singular value decomposition (SVD) of $\boldsymbol{F}$ has singular values $255,14.858$, and 0.8003 . This shows that any image formed from $\boldsymbol{F}$ will be approximately rank-2. Of course, usually we do not know $\boldsymbol{F}$. But the SVD for the collection of color vectors $\rho$ similarly shows a reduced rank - singular values are $9093,349,29$, i.e. 
approximately a scaling of those for $\boldsymbol{F}$. Fig.1(c,d) show two views of the RGB values plotted in colorspace: colors lie within an elliptical region on a fairly flat surface. 1

\subsection{Two-dimensional color-space}

To find the color-space plane we use a robust Least Median of Squares (LMS) regression [16]; outliers that do not lie in the plane are automatically identified. To find that vector $v_{3}$ to which most vectors $\rho$ are orthogonal, suppose that $v_{3}$ has third component 1 . Now form the $N \times 3$ collection $\mathcal{R}$ of vectors $\rho$, with $N$ the number of pixels. The set of equations we wish to approximate is: the $N \times 2$ matrix formed from the first two columns of $\mathcal{R}$ times an unknown 2-vector, equals the $N \times 1$ array given by the third column of $\mathcal{R}$.

The LMS regression defines a robust dispersion estimate in this case equal to

$$
s_{0}=k\left(1+5 /(N-2) \sqrt{\operatorname{med} r_{i}^{2}}\right)
$$

where $r_{i}$ is the residual for the $i^{\text {th }}$ case and $k \sim 14826$. Then an RGB point is accepted as corresponding to the model if $\left|r_{i} / s_{0}\right| \leq 2.5$; else the point is an outlier and is rejected. The robust coefficient of determination $\mathrm{R}^{2}$ is 0.9998 for this regression applied to Fig. $1(\mathrm{c}, \mathrm{d})$. Therefore one can identify outliers for this regression, and Fig.2(a) shows the mask of outliers for this image. Outliers make up $13 \%$ of non-background pixels.

To erect a 2-dimensional coordinate system on this plane we form projections of vectors $\rho$ onto the plane orthogonal to $v_{3}$

$$
\rho^{\perp}=\rho-\left(\rho \cdot v_{3}\right) v_{3}
$$

and then calculate the SVD of these projections, resulting in two more basis vectors $\boldsymbol{v}_{1}$ and $\boldsymbol{v}_{2}$. Now form orthogonal matrix $\boldsymbol{V}$ with columns equal to vectors $v_{1}, v_{2}, v_{3}$. Two-component vectors in the colorspace plane correspond to projections onto the first two columns of $\boldsymbol{V}$. Denote by $\boldsymbol{V}^{\prime}$ this $3 \times 2$ matrix, and form a set of 2-component colors via

$$
\mathcal{R}^{\prime}=\mathcal{R} \boldsymbol{V}^{\prime}
$$

with $\mathcal{R}^{\prime}$ an $N \times 2$ array comprised of 2-component vectors $\rho^{\prime}$. Fig.2(b) shows a scaled scatterplot of the components of $\rho^{\prime}$ values in the color-space plane.

\subsection{Ellipse boundary: Theory}

Let us rewrite eqn.(4) explicitly in terms of the individual columns $\boldsymbol{f}_{i}$ of matrix $\boldsymbol{F}$ as

$$
\rho^{T}=n^{T}\left(f_{1} f_{2} f_{3}\right) \text {. }
$$

Inliers for the three RGB image channels encode the shading field for the three effective color-strengthdirection vectors $\boldsymbol{f}_{i}$. If these three lights $\boldsymbol{f}_{i}$ were linearly independent, then we would indeed see an ellipsoid in color space. Instead, we see a 2-dimensional set of vectors

$$
\mathcal{R}^{\prime}=\mathcal{R} \boldsymbol{V}^{\prime}=(\mathcal{N} \boldsymbol{F}) \boldsymbol{V}^{\prime}=\mathcal{N}\left(\boldsymbol{F} \boldsymbol{V}^{\prime}\right) \equiv \mathcal{N} \boldsymbol{F}^{\prime}
$$

with $\boldsymbol{F}^{\prime}$ a $3 \times 2$ matrix with 3 -vector columns

$$
f_{i}^{\prime}, i=1 . .2: \quad F^{\prime}=\left(f_{1}^{\prime} f_{2}^{\prime}\right) \text {. }
$$

For any pixel, the projected 2 -vector is

$$
\rho^{\prime T}=n^{T} \boldsymbol{F}^{\prime} \text {. }
$$

The observed set of $\rho^{\prime}$ can be thought of as the projections of the shell of an ellipsoid onto the $v_{1}-v_{2}$ plane. They satisify the following

\footnotetext{
${ }^{1}$ The axes should actually be at the bottom of the data points: RGB values form a filled ellipse centered on the origin. Values fall away from the elliptical boundary near the origin because points that see fewer lights are darker.
}

Theorem: The ellipse boundary for a set of vectors $\rho^{\prime}$ is given by the quadratic form $C^{\prime}$, with

$$
\begin{gathered}
\boldsymbol{\rho}^{\prime T} \boldsymbol{C}^{\prime} \boldsymbol{\rho}^{\prime}=1, \quad \boldsymbol{C}^{\prime}=\left(\boldsymbol{F}^{\prime+}\right)\left(\boldsymbol{F}^{\prime+}\right)^{T}, \\
\boldsymbol{F}^{\prime+}=\left(\boldsymbol{F}^{\prime T} \boldsymbol{F}^{\prime}\right)^{-1} \boldsymbol{F}^{\prime T}
\end{gathered}
$$

The proof is given in [5]. The ellipse $\rho^{\prime T} C^{\prime} \rho^{\prime}=1$ forms the boundary of the filled ellipse. Some algebra shows that $\left(C^{\prime}\right)_{i i}^{-1}=\kappa_{i} \equiv\left\|f_{i}^{\prime}\right\|^{2}, i=1 . .2,\left(C^{\prime}\right)_{12}^{-1}=$ $\left(C^{\prime}\right)_{21}^{-1}=\mu \equiv f_{1}^{\prime} \cdot f_{2}^{\prime}$

\subsection{Ellipse boundary: Recovery}

Since the plot of Fig.2(b) is convex we form the convex hull of $\rho^{\prime}$ values as the set of perimeter points; we add the negatives of $2 \mathrm{D}$ colors $\rho^{\prime}$ and again use the LMS regression. Fig.2(b) also shows the convex hull along with the recovered and correct ellipse boundary. $\mathrm{R}^{2}$ is very close to unity.

\section{From 2 to 3 Dimensions}

\subsection{Relation between $\mathrm{F}^{\prime}$ and $\mathrm{F}$ \\ The 2-space color matrix $\boldsymbol{F}^{\prime}$ is given by}

$$
\boldsymbol{F}^{\prime}=\boldsymbol{F} \boldsymbol{V}^{\prime} \text {. }
$$

If $\boldsymbol{V}$ were actually the SVD eigenvectors for $\boldsymbol{F}$, instead of being recovered from the $\rho$, an approximately rank2 matrix $\boldsymbol{F}$ could be restored from the $3 \times 2$ matrix $\boldsymbol{F}^{\prime}$ via $\quad \boldsymbol{F} \simeq \boldsymbol{F}^{\prime} \boldsymbol{V}^{\prime T}$.

Under what conditions is the $\boldsymbol{V}$ recovered from the $\rho$ values equal to that for matrix $\boldsymbol{F}$ ? Using least squares, eigenvectors resulting from the regression $\rho \simeq$ $c_{i} v^{i}$ over $I=1 . . N$ pixels satisfy

$$
\sum_{I=1}^{N} \sum_{k=1}^{3} \rho_{m}^{I} \rho_{k}^{I} v_{k}^{i}=\lambda^{i} v_{m}^{i}
$$

Now substituting $\rho_{k}^{I}=\sum_{j=1}^{3} n_{j}^{I} F_{j k}$ we have

$$
\sum_{j} \sum_{p} \sum_{k} K_{j p} F_{j m} F_{p k} v_{k}^{i}=\lambda^{i} v_{m}^{i}
$$

with $K_{j p}$ the raw cross-product matrix of normal vectors $K_{j p}=\sum_{I=1}^{N} n_{j}^{I} n_{p}^{I}$. Eqn.(17) says that vectors $\boldsymbol{v}^{i}$, derived from the $\rho$, are the same vectors that would be found for matrix $\boldsymbol{F}$ provided the (non-meansubtracted) $3 \times 3$ covariance matrix $K_{j p}$ equals a diagonal matrix. This is in fact close to true for the normals making up Fig.1(a).

\subsection{Recovering $\mathrm{F}^{\prime}$}

If we could find the two 3 -vectors $f_{1}^{\prime}, f_{2}^{\prime}$ making up $\boldsymbol{F}^{\prime}$ then the two images $\rho_{1}^{\prime}, \rho_{2}^{\prime}$ could be used in 2image photometric stereo using $f_{1}^{\prime}, f_{2}^{\prime}$ as the "lights". So far, our robust regression of the ellipse boundary has provided three of the six unknowns in $\boldsymbol{F}^{\prime}, v i z ., \kappa_{1}$, $\kappa_{2}$, and $\mu$. However, from $\$ 4.1$ we know that the 3 dimensional $\boldsymbol{F}$ is given in terms of $\boldsymbol{f}_{1}^{\prime}, \boldsymbol{f}_{2}^{\prime}$ via matrix $V^{\prime}$. But from (4a) it is easy to show that $\left(\rho_{1}, \rho_{2}, \rho_{3}\right)$ are linearly dependent. Therefore one would suppose that there would be nothing further to gain by reexamining the RGB images, rather than those for $\rho_{1}^{\prime}$, $\rho_{2}^{\prime}$. This turns out to be not the case.

Since we know the lengths of $\boldsymbol{f}_{1}^{\prime}, \boldsymbol{f}_{2}^{\prime}$ from $\kappa_{1}, \kappa_{2}$, we seek the tilts $\tau_{1}^{\prime}, \tau_{2}^{\prime}$ and slants $\sigma_{1}^{\prime}, \sigma_{2}^{\prime}$ for normalized vectors $\tilde{\boldsymbol{f}}_{1}^{\prime}, \tilde{\boldsymbol{f}}_{2}^{\prime}$, We could employ a graylevel sourcedirection-finder to find the tilt and slant for 3 -vectors $f_{i}, i=1 . .3$ from the RGB images, but in practice 
slants derived from such estimators are quite inaccurate [7]. However, tilt estimations from such methods can be fairly reliable. In fact, the recent extension of the Zheng and Chellappa tilt voting method [17] performs remarkably well.

Since the tilts for the three RGB images are related nonlinearly to the tilts and slants for the $2 \mathrm{D}$ images, the three observations of tilts in 3D yield three additional constraints on $\boldsymbol{F}^{\prime}$. To see this, let us write out $\boldsymbol{F}=\boldsymbol{F}^{\prime} \boldsymbol{V}^{\prime}$ explicitly in terms of estimated $T_{i} \equiv \tan \left(\tau_{i}\right), i=1 . .3$, and unknown components of $f_{i}^{\prime}, i=1 . .2$. Let the two unknown 3 -vectors $f_{i}^{\prime}$ have components $f_{k}^{\prime i}$ and let the three $\boldsymbol{f}_{j}$ have components $f_{k}^{j}$. Then the tilt equations in 3 dimensions are

$$
f_{1}^{1} T_{1}=f_{2}^{1}, f_{1}^{2} T_{2}=f_{2}^{2}, f_{1}^{3} T_{3} f_{2}^{3} .
$$

Translating vectors $f$ into vectors $f^{\prime}$ via (15) and regrouping, we have

$$
\left(\begin{array}{llll}
T_{1} r & T_{1} u & -r & -u \\
T_{2} s & T_{2} v & -s & -v \\
T_{3} t & T_{3} w & -t & -w
\end{array}\right)\left(f_{1}^{\prime 1}, f_{1}^{\prime 2}, f_{2}^{\prime 1}, f_{2}^{\prime 2}\right)^{T}=0
$$

where $r=v_{1}^{1}, s=v_{2}^{1}, t=v_{3}^{1} ; u=v_{1}^{2}, v=v_{2}^{2}, w=v_{3}^{2}$. It is clear that the matrix in (19a) is of rank 3 , if $T_{1}, T_{2}, T_{3}$ are all different. Even if the rank of $\boldsymbol{F}$ were exactly 2 , with each column of $\boldsymbol{F}$ a linear combination of the other two columns, the matrix above is still rank-3.

Therefore tilt estimations in RGB images provide three independent equations for determining the four unknowns in vectors $\tilde{\boldsymbol{f}}_{1}^{\prime}, \tilde{\boldsymbol{f}}_{2}^{\prime}$; the final equation $\mu=$ $f_{1}^{\prime} \cdot f_{2}^{\prime}$ provides the fourth constraint, viz.

$$
\mu /\left(\kappa_{1} \kappa_{2}\right)=\tilde{f}_{1}^{\prime 1} \tilde{f}_{1}^{\prime 2}+\tilde{f}_{2}^{\prime 1} \tilde{f}_{2}^{\prime 2}
$$

$$
+\left[1-\left(\tilde{f}_{1}^{\prime 1}\right)^{2}-\left(\tilde{f}_{2}^{\prime 1}\right)^{2}\right]^{1 / 2}\left[1-\left(\tilde{f}_{1}^{\prime 2}\right)^{2}-\left(\tilde{f}_{2}^{\prime 2}\right)^{2}\right]^{1 / 2}
$$

Note that the third component of the $f_{i}^{\prime}$ can be taken positive [5].

Eqns. (19a, 19b) in four unknowns reduces to a quadratic equation in the square of one component. Of the two possible solutions, only one produces the correct, input, 3D tilt angles. The other solution has lights reversed in the $x-y$ plane.

Values found for the $f^{\prime}$ vectors are as follows: $f_{1}^{\prime}$ : $\sqrt{\kappa_{1}}=255.022, \tau=155.235, \sigma=10.176, f_{2}^{\prime}: \sqrt{\kappa_{2}}=$ $14.858, \tau=-20.142, \sigma=76.190$. For comparison, the correct values are $\boldsymbol{f}_{1}^{\prime}: \sqrt{\kappa_{1}}=255.022, \tau=154.978, \sigma=$ $6.121, f_{2}^{\prime}: \sqrt{\kappa_{2}}=14.858, \tau=-18.841, \sigma=83.914$. As can be seen, the method does very well.

\subsection{Recovery of normals}

Once "lighting" vectors are recovered, the situation is essentially identical to 2-image photometric stereo, with two known lights $[12,11]$. One forms an orthonormal basis $\tilde{a}_{i}, i=1 . .3$ from vectors $f_{j}^{\prime}, j=1 . .2$ and their cross-product, and then writes the normal in terms of this basis (see [5]). The resulting quadratic equation yields

$$
\boldsymbol{n} \cdot \tilde{\boldsymbol{a}}_{3}= \pm \sqrt{1-\left(\boldsymbol{n} \cdot \tilde{a}_{1}\right)^{2}-\left(\boldsymbol{n} \cdot \tilde{a}_{2}\right)^{2}}
$$

The problem thus becomes one of determining the correct sign at each pixel. The solution is ostensibly as follows: first identify those points where the component $\boldsymbol{n} \cdot \tilde{\boldsymbol{a}}_{3}$ is zero. Then within regions bounded by curves of such points first assign the positive, and then the negative sign. The correct sign is that which most closely obeys an integrability condition summed over the region.

However, carrying out such a scheme for a nonsmooth surface such as Fig.1(a) is problematic. This part of the overall problem is not completely addressed here, in that the reduction of orientation-from-color to photometric stereo is the objective. Nonetheless in $\$ 5$ we set out a new scheme for covering the image with the correct sign, for smooth surfaces.

For our exemplar image, however, there are difficulties in finding curves of zero third component. Fig.3(a) shows the value of the correct $\operatorname{sign}$ for $\boldsymbol{n} \cdot \widetilde{\boldsymbol{a}}_{3}$. To arrive at this answer we have at our disposal the image of the absolute value of this quantity. Low values in this absolute value should correspond to areas where the sign changes in Fig.3(a). Fig.3(b) shows an image, for non-outlier pixels, of values of $\left|\boldsymbol{n} \cdot \tilde{\boldsymbol{a}}_{3}\right|$ less than $20 \%$ of the maximum value. One can see that it is difficult to extract Fig.3(a) from Fig.3(b). To underline the difficulty of the problem, consider Fig.3(c): this is the same as Fig.3(b) except correct values of surface normals and lights are used. Even from this best possible image it it difficult to extract the correct sign.

Nevertheless it is interesting to compare recovered normals using the correct sign. We cannot recover normals for points designated as outliers, and to explicitly reconstruct a depth map we must bridge such lacunae in the orientation information. Harris' coupled depthslope surface reconstruction method [8] provides one method to do so, but it is slow. Alternatively, we can simply use all the pixels, ignoring the issue of outliers. Fig. $3(\mathrm{~d})$ shows the reconstructed depth map using a simpler Poisson equation solution. The correlation between the depth map of Fig.1(a) and this one is 0.9036 . Thus, 2-image photometric stereo, derived from multilight orientation-from-color, works reasonably well if the correct sign is recoverable.

\section{Real Image}

Fig.4(a) show an RGB image of a (brown) egg (displayed in black and white). The egg was placed near a window on an overcast day. Other lighting came from a ceiling fluorescent room light.

\subsection{Regression results}

The scatterplot of RGB values in color space is again very close to planar, with robust regression $R^{2}=0.9896$. Fig.4(b) shows the mask of inlier points. The $2 \mathrm{D}$ plot of $\rho^{\prime}$ is shown in Fig.4(c), along with the fitted ellipse. Since the scatterplot is non-convex, we simply regress on the perimeter of the data cluster, marked $P$ in the figure; the regression can be improved by omitting perimeter points near the origin by removing data for the first quartile of $\rho_{1}^{\prime}$ values. For this regression $R^{2}$ is 0.9930 . A useful self-check is to make sure that most $\boldsymbol{\rho}^{\prime}$ points fall on or within the ellipse boundary given by the matrix $C^{\prime}$ produced by the regression. Here, essentially all inliers pixels are encompassed by the ellipse.

From matrix $C^{\prime}$, we get $\sqrt{\kappa_{1}}=314.13, \sqrt{\kappa_{2}}=$ 39.91 , and angle $60.53^{\circ}$ between vectors $\boldsymbol{f}_{1}^{\prime}, \boldsymbol{f}_{2}^{\prime}$. To estimate the tilt angles for Fig.4(a), we used Zheng and Chellappa's original tilt voting method [22] with results $58^{\circ}, 47^{\circ}, 43^{\circ}$ for the three images. Now we can 
solve the four equations $(19 \mathrm{a}, \mathrm{b})$ together for vectors $\boldsymbol{f}_{1}^{\prime}, \boldsymbol{f}_{2}^{\prime}$. Eqn.(15) gives $\boldsymbol{F}$ in terms of the $2 \mathrm{D}$ vectors; the results have tilts as above and slants $21^{\circ}, 30^{\circ}, 34^{\circ}$.

\subsection{Sign of third component}

Here we propose a simple method for identifying positive and negative regions of (20). Let us assume the existence of an occluding boundary in the image. On that boundary, we must have no component in the camera direction, $n_{3} \equiv 0$. Therefore

$$
n_{3}=\left(\boldsymbol{n} \cdot \tilde{\boldsymbol{a}}_{1}\right) \tilde{a}_{3}^{1}+\left(\boldsymbol{n} \cdot \tilde{\boldsymbol{a}}_{2}\right) \tilde{a}_{3}^{2}+\left(\boldsymbol{n} \cdot \tilde{\boldsymbol{a}}_{3}\right) \tilde{a}_{3}^{3}=0 \text {. }
$$

Since we know the sign of $\tilde{a}_{3}^{3}$, we can determine the sign of $\left(\boldsymbol{n} \cdot \tilde{\boldsymbol{a}}_{3}\right)$ on an occluding boundary. If clear signboundary curves ("separation" curves [21]) are found within the image by thresholding $\left|\left(\boldsymbol{n} \cdot \widetilde{\boldsymbol{a}}_{3}\right)\right|$, then the appropriate sign can be grown in from the occluding boundary. It is reasonable to assume that low values in the absolute value of $\left(\boldsymbol{n} \cdot \widetilde{\boldsymbol{a}}_{3}\right)$ arise from a zerocrossing of the sign.

Fig.4(d) is an image of $\left|\left(\boldsymbol{n} \cdot \tilde{\boldsymbol{a}}_{\mathbf{3}}\right)\right|$, with small values outlined. We can understand this image by considering the separation line for a 2:1:1 ellipsold, with respect to the $\tilde{a}_{3}$ direction: surface normals perpendicular to this direction lie on an arc, with the positive sign to be taken above the arc and the negative sign below.

For the egg image, the sign all along the top occluding boundary is positive. Thus we should grow the positive sign inwards from the boundary in the top part of the image. Since values of $\left|\left(\boldsymbol{n} \cdot \tilde{\boldsymbol{a}}_{3}\right)\right|$ are all low in the area delineated in Fig.4(d), we take the separation line to be the bottom border of the low $-\left|\left(\boldsymbol{n} \cdot \tilde{\boldsymbol{a}}_{3}\right)\right|$ area. Therefore, in eqn.(20), the sign is positive in the top area of the egg and negative in the bottom area. For this image, the correct sign coincides with the sign specified by the simple condition $n_{3} \geq 0$.

Figs.5(a-b) show synthetic images of the recovered normal vectors shaded from directions $(1,1,1)$, and $(-1,-1,1)$. One can see that the algorithm does a reasonable job of recovering the shape of the egg. Here we cannot recover normals for outlier points, excluded by the mask in Fig.4(b). But again using all pixels and ignoring the issue of outliers, we arrive at the reconstructed depth map of Fig.5(c) by using a Poisson equation solution.

\section{Conclusions}

The exemplar synthetic image of Fig. 1 shows that rank-reduced color space data can result even using a quite broad selection of typical illuminants. The egg image was taken using ordinary office lighting and is also rank-2; in fact, in test images we found that rank2 lighting is the rule, rather than the exception. Therefore some method of dealing with this type of lighting environment is crucial for a general purpose shapefrom-shading scheme to succeed. The present analysis sets out a simple method for recovering surface orientation from such images.

Of course, the method depends on the success of two sub-procedures - (a) 2-image photometric stereo; and (b) a light-source tilt finder - and could be improved by improved versions of these algorithms.

\section{References}

[1] M.J. Brooks and B.K.P. Horn. Shape and source from shading. In IJCAI, pp. 932-936, Aug. 1985.
[2] M.S. Drew. Direct solution of orientation-from-color problem using a modification of Pentland's light source direction estimator, 1995. Comp. Vis. \& Image Und To appear.

[3] M.S. Drew. Shape from color. Tech. Rept. CSS/LCCR TR 92-07, Simon Fraser Univ., 1992. Available using ftp://fas.sfu.ca/pub/cs/techreports/1992/ as CSSLCCR92-07.ps.Z.

[4] M.S. Drew. Robust specularity detection from a single multi-illuminant color image. CVGIP:Image Und., 59:320-327, 1994.

[5] M.S. Drew. 2 goes into 3: Reduction of rank reduced orientation from color problem with many unknown lights to two image known illuminant photometric stereo. Tech. Rept. CSS/LCCR TR 9508, Simon Fraser Univ., 1995. Available using ftp://fas.sfu.ca/pub/cs/techreports/1995/ as CSSLCCR95-08.ps.Z.

[6] M. S. Drew and L. L. Kontsevich. Closed-form attitude determination under spectrally varying illumination. In CVPR, pp. 985-990, 1994.

[7] D. Gibbins, M.J. Brooks, and W. Chojnacki. Light source direction from a single image: a performace analysis. Austral. Comp. J., 23:165-174, 1991.

[8] J. G. Harris. A new approach to surface reconstruction: the coupled depth/slope model. In ICCV, pp. 277-283, 1987.

[9] B. K. P. Horn. Robot Vision. MIT Press, 1986.

[10] L. L. Kontsevich, A. P. Petrov, and I. S. Vergelskaya. Reconstruction of shape from shading in color images. J. Opt. Soc. Am. A, 11:1047-1052, 1994.

[11] R. Kozera. On shape recovery from two shading patterns. Int. J. of Patt. Recog. and Artif. Intell., 6:673698, 1992.

[12] R. Onn and A. Bruckstein. Integrability disambiguates surface recovery in two-image photometric stereo. Int. J. of Comp. Vis., 5:105-113, 1990.

[13] A. P. Pentland. Finding the illuminant direction. $J$. Opt. Soc. Am. A, 72:448-455, 1982.

[14] A. P. Petrov. Color and Grassman-Cayley coordinates of shape. In B. E. Rogowitz, M. H. Brill, and J. P. Allebach, editors, pp. 342-352. SPIE vol. 1453, 1991.

[15] A. P. Petrov and L. L. Kontsevich. Properties of color images of surfaces under multiple illuminants. $J$. Opt. Soc. Am. $A, 11: 2745-2749,1994$.

[16] P. J. Rousseeuw and A. M. Leroy. Robust Regression and Outlier Detection. Wiley, 1987.

[17] H. Singh and R. Chellappa. An improved shape from shading algorithm. Tech. Rept. CAR-TR-700, Univ. of Maryland, 1994.

[18] R. J. Woodham. Photometric method for determining surface orientation from multiple images. Optical Eng., 19:139-144, 1980.

[19] R. J. Woodham. Gradient and curvature from the photometric-stereo method, including local confidence estimation. J. Opt. Soc. Am. A, 11:3050-3068, 1994.

[20] R. J. Woodham, Y. Iwahori, and R. A. Barman. Photometric stereo: Lambertian reflectance and light sources with unknown direction and strength. Tech. Rept. TR 91-18, Univ. of Brit. Columbia, 1991.

[21] J. Yang, N. Ohnishi, and N. Sugie. Two image photometric stereo method. pp. 452-463. SPIE Vol. 1826 , 1992.

[22] Q. Zheng and R. Chellappa. Estimation of illuminant direction, albedo, and shape from shading. IEEE Trans. Patt. Anal. and Mach. Intell., 13:680-702, 1991. 
(a)

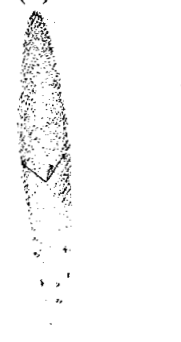

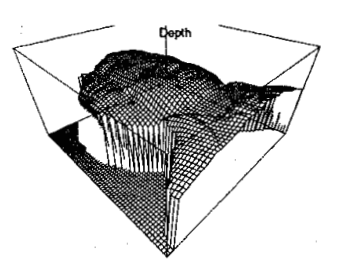

Figure 1: (a): Depth map of plaster bust. (b): Shaded

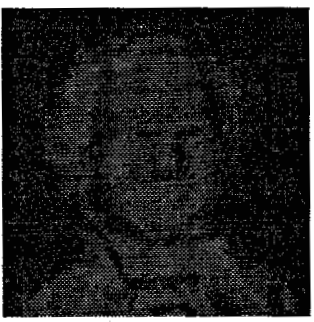

(b)

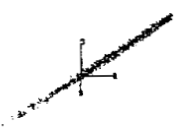

Figure 1: (a): (c) Depth map of plaster bust. (b): Shaded with five colored lights. $(c, d)$ : RGB points in color space: filled ellipse centered on origin.

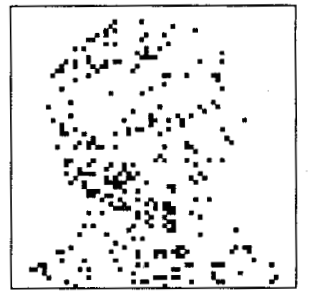

(a)

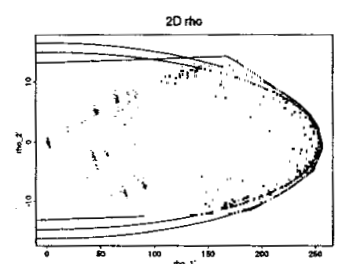

(b)
Figure 2: (a): Mask for inliers for robust regression to planar colors. (b): Colors in 2-dimensional plane, with robust fit to convex hull and correct ellipse.

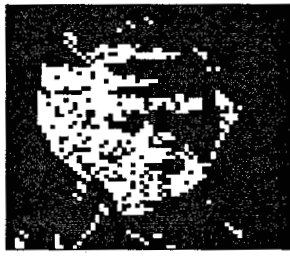

(a)

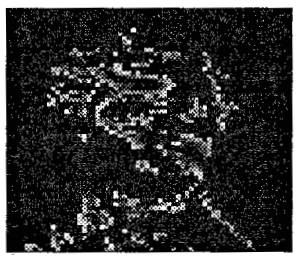

(c)

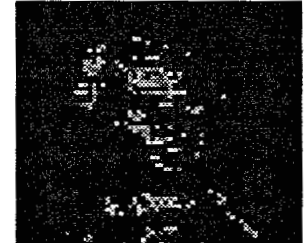

(b)

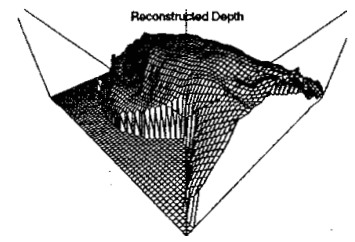

(d)
Figure 3: (a): Correct sign for component of normal in third direction. (b): Low values in absolute value of recovered third component. (c): Low values in correct absolute value. (d): Recovered depth map.

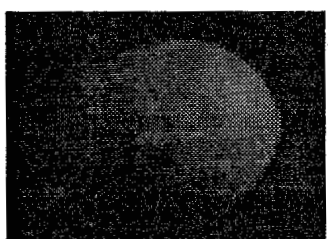

(a)

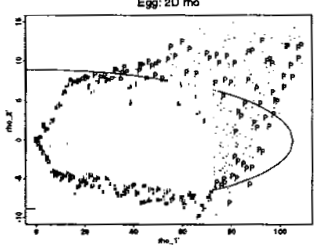

(c)

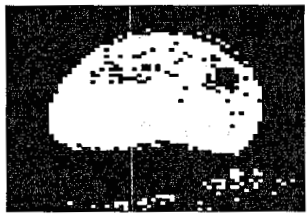

(b)

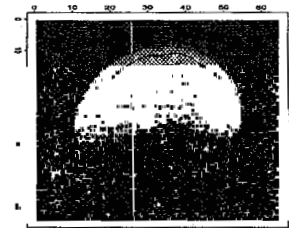

(d)
Figure 4: (a): RGB image. (b): Inlier mask for regression to planar colors. (c): 2D colors and robust regression to perimeter points. (d): Absolute value of third component in planar basis; low value region is delineated.

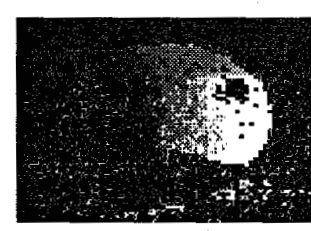

(a)

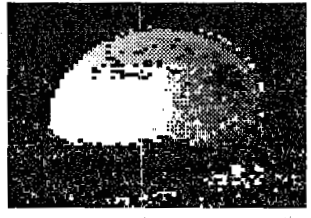

(b)

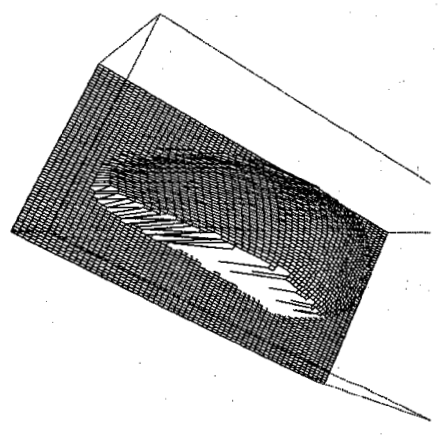

(c)

Figure 5: $(a, b)$ : Recovered normals, shaded from $(1,1,1)$, and $(-1,-1,1)$. (c): Depth map recovered from Poisson equation (using outlier pixels as well as inliers). 